\title{
Que peut faire la santé publique pour régler les injustices liées aux maladies infectieuses?
}

\author{
Moloughney BW ${ }^{1,2 *}$
}

\section{Résumé}

Contexte : La reconnaissance de l'importance des conditions sociales permet d'éclairer les interventions précoces en santé publique liées aux épidémies de maladies infectieuses. En influençant l'exposition, la vulnérabilité et l'accès aux services de santé, les déterminants sociaux de la santé continuent de causer des inégalités dans la répartition des maladies infectieuses. Ces inégalités évitables et injustes sont considérées comme des injustices.

Analyse : Un certain nombre de défis et d'obstacles empêchent l'adoption plus vaste de mesures de santé publique visant à lutter contre les déterminants sociaux de la santé et les injustices, y compris un manque de clarté sur ce que la santé publique devrait ou pourrait faire. Le Centre de collaboration nationale des déterminants de la santé (CCNDS) a défini quatre rôles principaux quant aux mesures de santé publique visant les déterminants sociaux de la santé et les injustices. Le présent document décrit ces rôles et comprend des exemples de leur application au domaine des maladies infectieuses. II souligne également la contribution précieuse apportée par les organisations, qui jouent un rôle de leaders et de soutien pour que les représentants des programmes et les employés continuent de prendre des mesures relativement aux déterminants sociaux de la santé et aux injustices.

Conclusion : Bien que le défi soit vaste et complexe, les approches comme la définition des rôles du CCNDS pour l'adoption de mesures de santé publique permettent de dresser une liste d'options qui facilite l'analyse et la prise de mesures visant à lutter contre les déterminants sociaux de la santé et les injustices liées aux maladies infectieuses.

\section{Affiliations}

'BWM Health Consultants Inc., Newmarket (Ontario)

${ }^{2}$ Dalla Lana School of Public Health, Université de Toronto, Toronto (Ontario)

${ }^{\star}$ Correspondance : brent. moloughney@rogers.com

Citation proposée : Moloughney BW. Que peut faire la santé publique pour régler les injustices liées aux maladies infectieuses? Relevé des maladies transmissibles au Canada 2016;42-Suppl 1:S16-20. https://doi.org/10.14745/ccdr.v42is1a03f

\section{Introduction}

La santé publique moderne a vu le jour en réponse aux épidémies qui sont survenues après le début de la révolution industrielle. La migration de masse vers les villes, les mauvaises conditions de vie résultant du surpeuplement et des logements mal ventilés, l'eau potable contaminée et l'élimination inconsidérée des eaux usées ont causé la propagation de maladies infectieuses. Les différences marquées du taux de mortalité entre les groupes de population ont déclenché un mouvement de défense des droits visant l'adoption de mesures sanitaires pour améliorer les conditions de vie des pauvres (1).

De nos jours, ces conditions sont appelées les « déterminants sociaux de la santé " et sont définis comme étant les "facteurs sociaux, politiques et économiques interdépendants qui créent les conditions dans lesquelles les gens vivent, apprennent, travaillent et jouent » (2). Les injustices en matière de santé sont, pour leur part, des inégalités en matière de santé associées à des désavantages sociaux injustes et susceptibles d'être modifiés (2). De la Charte d'Ottawa pour la promotion de la santé (3) au Rapport sur la santé des Canadiens et des Canadiennes (4) et aux contributions à la Commission des déterminants sociaux de la santé de l'Organisation mondiale de la Santé (5), le Canada a mis l'accent sur l'importance des déterminants de la santé et des injustices à l'échelle internationale. Néanmoins, il se peut que les professionnels de la santé et les organisations de santé publique trouvent difficile de savoir exactement ce qu'ils devraient et ce qu'ils peuvent faire pour lutter contre les injustices (6). Tout en reconnaissant la complexité du problème et les obstacles associés au fait de s'attaquer aux questions d'équité en matière de santé, le présent document décrit un ensemble de rôles de santé publique qui devraient être adoptés si l'on veut lutter contre les injustices dans le contexte des maladies infectieuses.

\section{Analyse}

La distinction entre les inégalités et les injustices en matière de santé est importante. Les inégalités en matière de santé sont les différences mesurables sur le plan de la santé entre les personnes, les groupes ou les collectivités (2). Par exemple, le taux de la maladie $X$ dans une population $Y$ diffère de son taux dans une population $\mathrm{Z}$.

Tel qu'il a été défini plus tôt, les injustices en matière de santé sont des inégalités liées à des différences résultant de désavantages sociaux modifiables que de nombreuses personnes jugent inéquitables (2). Le concept de l'équité en matière de santé est lié à un engagement envers la justice sociale et les 
droits de la personne fondamentaux, comme celui de l'accès à de l'eau potable, à de la nourriture, à l'éducation et à des soins de santé (7).

Une façon d'envisager la manière dont les déterminants sociaux de la santé influent sur la maladie est de considérer comment ils influent sur l'exposition, la vulnérabilité et l'accès aux services de santé (8). Les déterminants sociaux de la santé ont une incidence sur ces trois aspects dans le cas de la tuberculose, par exemple: ils augmentent le risque d'exposition lorsque les gens vivent dans des logements surpeuplés et mal ventilés; et ils augmentent la vulnérabilité à l'infection lorsque l'apport nutritionnel est inadéquat; ils diminuent l'accès aux services de santé (9). Même dans un pays comme le Canada, qui a mis en place un programme universel de soins de santé, les problèmes d'accès comprennent la disponibilité des fournisseurs de soins de santé et de l'équipement de diagnostic dans les régions éloignées; les obstacles liés au paiement pour certaines classes de demandeurs réfugiés; les coûts de transport et ceux associés aux heures de travail manquées pour se rendre à des rendez-vous; et le niveau de confiance envers les professionnels de la santé (10).

Bien que la tuberculose ne soit plus courante au Canada, le taux d'incidence de la tuberculose active chez les Autochtones nés au Canada est 34 fois plus élevé que chez Canadiens non autochtones (11). Comme la tuberculose peut être évitée et guérie, les inégalités afférentes aux taux $d$ 'incidence de cette maladie chez les Autochtones reflètent une injustice en matière de santé. Il existe également des injustices liées à la tuberculose dans le cas des personnes nées à l'étranger. Par exemple, l'ampleur des obstacles associés aux déterminants sociaux de la santé pour l'accès aux soins de santé chez cette population a poussé le bureau de santé publique de Toronto à embaucher un travailleur social pour son programme de lutte contre la tuberculose pour traiter des questions de logement, de revenu et d'immigration (10).

L'analyse de l'incidence des déterminants sociaux de la santé sur l'exposition, la vulnérabilité et l'accès aux services de santé s'applique à d'autres maladies infectieuses. La compréhension ne garantit toutefois pas l'efficacité des mesures prises. Une analyse du contexte menée par le Centre de collaboration nationale des déterminants de la santé (CCNDS) en 2010 a révélé qu'il existe de nombreux obstacles à une prise de mesures de santé publique plus répandues pour lutter contre les déterminants sociaux de la santé et les injustices (6):

- le manque de clarté sur ce que la santé publique devrait ou pourrait faire;

- les données probantes limitées sur ce qui fonctionne pour atténuer les effets des déterminants sociaux de la santé sur la santé;

- les préoccupations concernant les approches axées sur le comportement et le mode de vie;

- les caractéristiques bureaucratiques de l'organisation;

- les limites de la capacité organisationnelle;

- le besoin de leadership, d'une communication plus efficace et de contextes politiques favorables.
L'une des principales conclusions de l'analyse du CCNDS était que, bien que les analyses et les mesures liées aux déterminants de la santé et aux injustices ne soient pas nouvelles dans le domaine de la santé publique, les approches adoptées pour lutter contre ceux-ci n'ont jamais été institutionnalisées ou ont été abandonnées en raison d'autres types de pressions (6). Pour régler ce problème, le CCNDS a recommandé que l'on définisse quatre rôles principaux de la santé publique, que le centre a adoptés pour orienter son travail de transfert de connaissances visant à faire progresser les mesures liées aux déterminants sociaux de la santé et l'équité en matière de santé grâce aux pratiques et aux politiques de santé publique (12). Ces rôles sont décrits ci-dessous.

\section{Évaluer l'existence et les répercussions des injustices en matière de santé ainsi que des stratégies efficaces pour les réduire, et en faire rapport}

Le rôle de l'évaluation et des rapports sur les injustices en matière de santé et sur les stratégies efficaces pour réduire leur prévalence et leurs répercussions incombe le plus naturellement aux organisations de santé publique et est essentiel si l'on veut étayer les mesures prises dans le contexte des trois autres rôles. De récents exemples appliqués au domaine des maladies infectieuses comprennent, sans y être limités, le rapport de 2013 de l'administrateur en chef de la santé publique du Canada, qui fait ressortir les inégalités liées à la tuberculose (11), ainsi que le rapport de la ville de Toronto qui souligne les injustices au chapitre des taux de chlamydiose chez les jeunes de cette ville (13).

Ces deux rapports contiennent des directives de haut niveau quant à la manière d'établir des étapes pour répondre aux injustices observées. Au cours de l'analyse du contexte menée par le CCNDS, bon nombre de répondants clés ont indiqué que la santé publique doit aller au-delà de la simple description des injustices et prendre des mesures pour y remédier (6), ce qui constitue l'objectif des trois rôles suivants.

\section{Modifier et orienter les interventions et les services pour aider à réduire les injustices, avec une compréhension des besoins uniques des populations qui subissent la marginalisation}

La compréhension des besoins uniques de populations concerne le fait de veiller à ce que la planification des interventions et des services en matière de santé publique considère les injustices et y réponde. L'une des approches de plus en plus fréquemment adoptées consiste, pour les organisations, à intégrer des évaluations de l'équité dans leur cycle de planification opérationnelle (14). Un excellent exemple de cette pratique est la vérification menée par Saskatoon Population and Public Health de son programme d'immunisation, qui a permis de déceler des différences marquées entre voisinages dans les taux de couverture vaccinale, les taux d'immunisation les plus faibles étant observés chez les voisinages affichant les plus faibles revenus (15). Des changements de pratique subséquents, y compris la redistribution des ressources du programme et le recours à des rappels pour les parents ou le personnel de garde, ont été associés à des réductions des différences dans les taux de couverture vaccinale chez les différents voisinages (16). 
Établir des partenariats avec d'autres organisations gouvernementales ou communautaires pour relever des façons d'améliorer les résultats sur la santé chez des populations qui subissent à la marginalisation

En raison des interdépendances complexes des déterminants sociaux de la santé, bon nombre d'organisations, outre celles qui œuvrent dans le domaine de la santé publique, travaillent pour répondre aux besoins de populations particulières. Néanmoins, en raison de la diversité des mandats et des objectifs, ainsi que de l'omniprésence des déterminants sociaux de la santé, les obstacles et les lacunes afférents aux services persistent. Une meilleure coordination et une planification conjointe par les acteurs au sein du système sont nécessaires si l'on veut éviter ces lacunes et y remédier. Par exemple, au Manitoba, une table ronde tripartite a été mise sur pied pendant et après la pandémie due au virus H1N1. À celle-ci participaient le gouvernement provincial, le gouvernement fédéral, l'Assembly of Manitoba Chiefs, la Manitoba Metis Federation Inc. et des représentants d'autorités sanitaires régionales, qui, tous, se sont efforcés d'améliorer la communication et la coordination entre les parties et ont réussi à atteindre un consensus sur bon nombre de questions (17). De tels partenariats devraient nous mener à une prestation de services plus efficace et plus efficiente.

Diriger, soutenir et participer, avec d'autres organisations, à I'analyse et à l'élaboration de politiques, ainsi qu'à la défense des droits pour une amélioration des déterminants de la santé et des questions de justice

La participation à l'élaboration de politiques et à la défense des droits est un rôle clé pour les organisations de santé publique, car un changement structurel des déterminants sociaux de la santé a des répercussions potentielles des plus importantes sur la santé de la population (18). Les politiques peuvent cibler une ou plusieurs des incidences de déterminants sociaux de la santé sur l'exposition, la vulnérabilité ou l'accès aux services. Par exemple, le rapport du dirigeant de santé de la province de la Colombie-Britannique, intitulé HIV, Stigma and Society: Tackling a Complex Epidemic and Renewing HIV Prevention of Gay and Bisexual Men in British Columbia (19) identifie plusieurs sous-populations qui sont plus vulnérables en raison de facteurs sociaux, politiques et environnementaux. La stigmatisation, le harcèlement verbal et la violence entraînent divers risques pour la santé, notamment davantage de comportements sexuels à risque et des obstacles plus importants à l'accès à des soins de santé appropriés, ainsi qu'une plus forte probabilité de piètres résultats en matière de santé mentale. Le dirigeant provincial de la santé recommande d'améliorer les facteurs de protection comme une éducation de la santé en matière de sexualité complète et inclusive qui conteste la stigmatisation et améliore les compétences en matière de prise de décisions; I'amélioration et l'extension des soins de santé offerts aux personnes séropositives; progrès au chapitre du soutien aux services de santé mentale et aux services aux personnes qui consomment des substances; et la révision des lignes directrices afférentes aux poursuites qui concernent le VIH (19).

\section{Surmonter les obstacles à la prise de mesures}

Les rôles proposés par le CCNDS prennent en considération ce que les organisations de santé publique peuvent faire pour traiter la question des déterminants sociaux de la santé et les injustices en santé, qui comprennent également les maladies infectieuses. Malgré I'historique de la santé publique et toutes nos connaissances sur les déterminants sociaux de la santé et les injustices en santé, la perception selon laquelle la réponse aux déterminants sociaux de la santé et aux injustices exigerait du temps supplémentaire pour les organisations de santé publique représente un autre obstacle à la prise de mesures (6). Pour régler cette question, les administrations incorporent de plus en plus des attentes explicites dans leurs programmes et normes fondamentaux. Par exemple, les programmes provinciaux de santé publique suivants ont incorporé l'atténuation des injustices dans le mandat de leurs responsables d'activités de santé publique :

Programme national de santé publique du Québec (20)

- Parmi les principales stratégies figurent le développement communautaire et la participation à des mesures intersectorielles visant à promouvoir la santé et le soutien aux groupes vulnérables

A Framework for Core Functions in Public Health (C.-B.) (21)

- Le document comprend une « lentille » à travers laquelle on peut évaluer les injustices en matière de santé et y répondre

- II relève huit manières dont les organisations de santé publique pourraient contribuer à réduire les inégalités en matière de santé

Normes de santé publique de l'Ontario (22)

- Le document établit que le traitement des déterminants de la santé et l'allégement des injustices sont des aspects fondamentaux du travail de santé publique

- L'une des exigences clés est d'identifier les populations locales prioritaires et de travailler auprès d'elles

Normes de santé publique de la Nouvelle-Écosse (23)

- Le document incorpore les quatre rôles définis par le CCNDS (évaluer l'existence et les répercussions des injustices en matière de santé ainsi que des stratégies efficaces pour les réduire, et en faire rapport; modifier et orienter les interventions et les services pour aider à réduire les injustices, avec une compréhension des besoins uniques des populations qui font face à la marginalisation; établir des partenariats avec d'autres organisations gouvernementales ou communautaires pour relever des façons d'améliorer les résultats sur la santé chez des populations qui font face à la marginalisation; surmonter les obstacles à la prise de mesures) au sein d'une même norme générale. 
Lorsque les normes de santé publique de la Nouvelle-Écosse ont été élaborées, on a relevé un défi concret auquel est confronté le personnel chargé de la prévention et du contrôle des maladies infectieuses lorsqu'il veut étendre son action aux déterminants sociaux de la santé et aux injustices. Tandis que les professionnels de la prévention et du contrôle des maladies infectieuses possèdent des connaissances fondamentales et s'appuient sur des intuitions pour étayer la compréhension et la prise de mesures afférentes aux déterminants sociaux de la santé qui entraînent des résultats néfastes sur la santé, les demandes opérationnelles associées aux responsabilités quotidiennes limitent le temps disponible pour mettre en œuvre de telles mesures. L'une des approches proposées était, pour les organisations, de soutenir ce personnel en contribuant aux initiatives liées aux déterminants sociaux de la santé, tandis qu'un personnel d'un autre secteur de l'organisation, comme des agents de promotion de la santé, pourrait se consacrer exclusivement à la mise en œuvre de mesures à plus long terme destinées à influer sur ces déterminants par l'entremise de politiques publiques plus saines et d'autres stratégies.

\section{Conclusion}

La prise de mesures sur les déterminants sociaux de la santé et pour répondre aux injustices fait partie intégrante des pratiques de la santé publique. De telles mesures sont reflétées dans I'historique de la santé publique qui sont décrites dans des rapports de référence qui remontent à des décennies et sont explicitement incluses dans des programmes de base. Bien qu'un certain nombre d'obstacles existent, les principaux rôles des organisations de santé publique pour la prise de mesures ont été relevés et appliqués aux maladies infectieuses et à d'autres types d'état de santé.

Tandis que les mesures concernant les déterminants sociaux de la santé et les injustices reposent sur les professionnels de la santé, les mesures de santé publique reflètent de façon prédominante les processus et les résultats au niveau organisationnel (6). Par exemple, la production d'un rapport qui souligne les injustices ou fait état de partenariats avec d'autres secteurs visant à mettre en œuvre des changements de politiques exige une décision délibérée au niveau organisationnel. Le fait de remplir I'un des quatre rôles définis par le CCNDS exige le leadership nécessaire pour établir des priorités, attribuer des ressources, façonner des comportements et des attitudes, éduquer le public et les décideurs et instaurer la capacité de l'organisation à entreprendre ce travail (6).

Au niveau systémique, il est également nécessaire que l'on fasse preuve de leadership et de bonnes habiletés de communication si l'on veut rendre les rôles et responsabilités en matière de santé publique explicites et offrir l'appui politique nécessaire à l'engagement et à la prise de mesures dans ce domaine. Bien que le défi soit vaste et complexe, les approches comme la définition des rôles du CCNDS pour l'adoption de mesures de santé publique permettent de dresser une liste d'options qui facilite l'analyse et la prise de mesures visant à lutter contre les déterminants sociaux de la santé et les injustices liées aux maladies infectieuses.

\section{Conflit d'intérêts}

Aucun.

\section{Références}

1. Susser M, Stein Z. The British Sanitary Movement: Edwin Chadwick. In: Susser M, Stein Z, editors. Eras in epidemiology: the evolution of ideas. New York: Oxford Scholarship Online; 2009. http://www.oxfordscholarship. com/view/10.1093/acprof:oso/9780195300666.001.0001/ acprof-9780195300666-chapter-6.

2. National Collaborating Centre for Determinants of Health. Glossary of essential health equity terms. Antigonish (NS): NCCDH, St. Francis Xavier University; 2014. (Disponible en français : http://nccdh.ca/images/uploads/Glossary_FR_ Feb_26.pdf).

3. World Health Organization; Health and Welfare Canada; Canadian Public Health Association. Ottawa Charter for Health Promotion. Geneva (CH): WHO; 1986.(Disponible en français : http://www.phac-aspc.gc.ca/ph-sp/docs/charterchartre/index-fra.php).

4. Federal Provincial and Territorial Advisory Committee on Population Health. Report on the Health of Canadians . Ottawa (ON): Health Canada; 1996. (Disponible en français : http://publications.gc.ca/collections/Collection/ H39-385-1996-1F.pdf).

5. Commission on Social Determinants of Health. Closing the gap in a generation: health equity through action on the social determinants of health. Geneva: World Health Organization; 2008. (Disponible en français : http://www. who.int/social_determinants/thecommission/finalreport/fr/).

6. National Collaborating Centre for Determinants of Health. Integrating social determinants of health and health equity into Canadian public health practice: environmental scan 2010. Antigonish (NS): National Collaborating Centre for Determinants of Health, St. Francis Xavier University; 2010. (Disponible en français : http://nccdh.ca/images/uploads/ Environ_Report_FR.pdf).

7. National Collaborating Centre for Determinants of Health. (2013). Let's talk: Health equity. Antigonish, NS: National Collaborating Centre for Determinants of Health, St. Francis Xavier University. (Disponible en français : http://nccdh.ca/ images/uploads/L\%E2\%80\%99\%C3\%A9quit\%C3\%A9_en_ sant\%C3\%A9_Parlons-en_2013.pdf).

8. Loring $B$, Robertson A. Obesity and inequities: guidance for addressing inequities in overweight and obesity. Copenhagen: WHO Regional Office for Europe; 2014. http://www.euro.who.int/__data/assets/pdf_ file/0003/247638/obesity-090514.pdf.

9. Hargreaves JR, Boccia D, Evans CA, Adato M, Petticrew M, Porter JD. The social determinants of tuberculosis: from evidence to action. Am J Public Health. 2011 Apr;101(4):654-62 
17. National Collaborating Centre for Determinants of Health. Learning from practice: equity in influenza prevention in Manitoba. Antigonish (NS): The Centre; 2015. (Disponible en français : http://nccdh.ca/images/uploads/Manitoba_FR_ Feb_20.pdf).

18. Frieden TR. A framework for public health action: the health impact pyramid. Am J Public Health. 2010 Apr;100(4):590-5.

19. British Columbia Provincial Health Officer. HIV, stigma and society: tackling a complex epidemic and renewing HIV prevention for gay and bisexual men in British Columbia. Victoria (BC): Ministry of Health; 2014. http://www2.gov. bc.ca/assets/gov/health/about-bc-s-health-care-system/ office-of-the-provincial-health-officer/reports-publications/ annual-reports/hiv-stigma-and-society.pdf.

20. Cardinal L, Francoeur D, Hamel M, Kirouac S, Théberge Y. Quebec Public Health Program 2003-2012. Quebec (QC): Ministère de la Santé et des Services sociaux; 2003. http://publications.msss.gouv.qc.ca/acrobat/f/ documentation/2003/03-216-02A.pdf.

21. Population Health and Wellness. A framework for core functions in public health: resource document. Victoria (BC): Ministry of Health Services; 2005. http://www.health.gov. bc.ca/library/publications/year/2005/core_functions.pdf.

22. Ontario Ministry of Health and Long-Term Care. Ontario public health standards. Toronto (ON): Queen's Printer for Ontario, 2008. (Disponible en français : http://health.gov. on.ca/fr/pro/programs/publichealth/oph_standards/docs/ ophs_2008f.pdf).

23. Nova Scotia Public Health. Nova Scotia Public Health Standards 2011-2016. Halifax (NS): Nova Scotia Public Health; 2011. (Disponible en français : http://novascotia. $\mathrm{ca} / \mathrm{dhw} /$ publichealth/documents/Public_Health_Standards_ FR.pdf).
16. Kershaw T, Cushon J, Dunlop T. Towards equity in immunization: the immunization reminders project. Saskatoon (SK): Saskatoon Health Region; 2011. http://www.saskatoonhealthregion.ca/locations_services/ Services/Health-Observatory/Documents/ReportsPublications/ImmRemindersProject_TwoPager_000.pdf. 